\title{
A history in the making?
}

\author{
Nikhil P. Hawal and Namratha Kulkarni
}

Department of Community Medicine, J.N.M.C; Belgaum, Karnataka

Rukhsar, an 18 month old girl from Howrah district, West-Bengal was the last reported case infected with Type-I polio virus. On $13^{\text {th }}$ January 2012, India surpassed one year without detecting a single case of wild polio virus. In 1988 when the Global Polio Eradication Initiative $(\mathrm{GPEI})^{3}$ was started, with the intensity of transmission experts believed that India would be the biggest hurdle owing to the sheer size and the diversity of the population and it would be the last country in the world to eradicate Polio. Despite the pessimistic prophesies of failure and almost fifty years after the successful work of Jonas Salk, India managed one entire year with no new cases of Polio. But celebrations will have to wait for another two years because for certificate of eradication two more years have to pass without a single case of Wild Polio Virus. In the seventies, World Health Organization (WHO) required one year declaring India free from small pox but in case of Polio the virus can remain dormant for a longer period of time, still the fact that no new cases of polio were from anywhere in India for one year is a matter of national pride.

Since the launch of the Global Polio Eradication Initiative in 1988, the incidence of wild poliovirus has reduced by 99 per cent - from 350,000 children paralyzed or killed annually in 125 endemic countries in 1988 to 620 cases reported in 16 countries in 2011 (as of 3 January, 2012) . $^{1}$. In 2006 , the number of polio-endemic countries (countries that have never stopped indigenous wild poliovirus transmission) was reduced to four - India, Nigeria, Pakistan and Afghanistan. With India's achievement the global effort now focuses on improving the implementation in Pakistan, Nigeria and Afghanistan. Tackling of the remaining 1 per cent remains the greatest challenge yet. ${ }^{5}$

\section{*Address for correspondence: nikhil Hawal \\ E-mail: hawnik1185@yahoo.in}

DOI: 10.5530/ijmedph.2.3.2
The road to this success was not easy. It needed a perfect blend of strong political will, flawless immunization strategies and most importantly undaunted determination of every Indian. Since 2000, the Type-I Wild Polio Virus showed cyclical trends every 4 years in 1998, 2002 and 2006 reported 648 cases of Type-I. The next outbreak in 2010 had to be stopped at all costs. The introduction of the bivalent oral polio vaccine (bOPV) came as a boon at a right time. From 741 cases in 2009 more than any other country in the world, India reported only 42 cases of the 1352 cases globally in 2010 and just one case in 2011. This is truly an extraordinary achievement. A study in The Lancet also pointed out that the bOPV was the most effective vaccine and could play a vital role in eradicating polio in the other countries.

\section{WHAT'S NEXT?}

We might have won the battle but the war against polio is far from over. The tag of zero cases in one year has brought with it a sense of complacency among the community and apparently the immunization program itself. We are now closer than ever and cannot afford to show any complacency for the next three years. In fact we need greater vigilance and have to work like polio is still lurking to attack us when least expected. We have to make sure every child continues to be vaccinated until global eradication is achieved because Polio anywhere is risk everywhere.

Another greatest challenge will now be to prevent any importation of the virus from neighboring countries of Pakistan and Afghanistan. Polio was a disease of the past in China until September 2011, 11 years after its eradication 10 new cases of WPV-1 and one death in China has led to millions of children in China being revaccinated. The genetic studies revealed that the virus had been imported from Pakistan; hence no matter how long a country has been polio-free, as long as global polio eradication has not yet been achieved, the risk for importation remains 
and constant vigilance is required. With overwhelming rates of infection, associated with overcrowding, poor sanitation and diarrheal diseases such an outbreak in India would be devastating.

Polio eradication will eventually require cessation of the OPV use from routine immunization otherwise the continued use will result in cases due to Vaccine associates paralytic polio (VAPP) and circulating vaccine derived polio virus (cVDPV). Since 2000 four polio outbreaks due to cVDPV have been reported in Philippines, Madagascar, China and Hispaniola., ${ }^{2,3}$ Genetically it is Sabin strain viruses that reverts back to the phenotypic and genotypic characters of a virulent strain and re-infects the children. A study by Helen Jenkins et al. from Imperial College London reaffirms that cVDPVs can regain transmissibility and pathogenicity that is similar to that of wild polioviruses. ${ }^{4}$ OPV use can also in rare cases cause paralytic polio. If the world wide OPV utilization patterns of 2005 continue after the confirmation of eradication it is estimated that there will be $250-500$ cases of VAPP each year and up to one polio outbreak due to cVDPV each year. ${ }^{3}$ Once the eradication of polio has been confirmed, the public health benefits of routine immunization with OPV may no longer outweigh the burden of the disease due to VAPP and VDPV and compromise the goal of polio free world. Apart from this, continued OPV use could rarely lead to prolonged excretion of VDPV from a child with severe immunodeficiency syndrome. Theoretically, these iVDPV could then reintroduce polio virus in the general population. In half a century of OPV use 28 cases of iVDPV have been documented, most of them from the high income countries. ${ }^{3}$ These could be the three main reasons for eventually stopping the use of OPV in routine immunizations, but the two main risks associated with OPV cessation will be immediate risk of cVDPV emergence and long term risk of polio virus re-introduction from a Vaccine manufacturing site, research facility or a diagnostic laboratory.

The best approach is to introduce IPV, achieve $90 \%$ coverage and only then stop OPV. This will be the challenge of the present generation. Dr. Ali Jafer Mohammed et al. from the Ministry of Health in Oman showed that fractional doses of IPV administered intradermally at 2, 4 and 6 months, as compared with full doses of IPV given intramuscularly in the same schedule, achieve similar seroconversion rates, though with lower antibody titers. ${ }^{7}$ The Global Polio Eradication Initiative is pursuing its work on evaluating a variety of approaches to achieve affordable IPV options for the post-OPV era. Despite the possible short comings in the future, currently OPV remains a key for global eradication to the point of simultaneous cessation across the globe. Until then the best possible thing to do will be to strengthen our AFP surveillance and detect any importations or cVDPV and maintain the momentum of our immunization programme.

The world is on the verge of history and today we are closer than ever. A remarkable success story has been the eradication of smallpox from the face of the earth. Is it possible to repeat the performance with reference to poliomyelitis? If the answer is "Yes", polio will be the second infectious disease to be eradicated. Rising cases in Pakistan and Nigeria and international spread of the virus (620 as on $3^{\text {rd }}$ Jan. 2012) are imminent threats to global eradication. With security and safety issues in Pakistan and Afghanistan and lack of political will in Nigeria, this could take six months or six years. The virus that originated in Nigeria, as a sense of moral justice will probably end there. Hope that history repeats itself and polio joins small pox as the second major disease to be eradicated from the face of the earth and we would be proud to be remembered as a part of this history in the making.

\section{REFERENCES}

1. WHO global action plan to minimize poliovirus facility-associated risk DRAFT - 2009.

2. WHO Frame work for National policy makers in OPV using countries.

3. WHO Cessation of routine Oral Polio Vaccine (OPV) use after global eradication.

4. Helen EJ, Bruce AR, Alex G, Christl AD, Michael M, Jukka C, et al. Implications of a Circulating VaccineDerived Poliovirus in Nigeria. N Engl J Med 2010; 362(25):2362-6.

5. John FM. The Bumpy Road to Polio Eradication. N Engl J Med 2010; 362(25):2346-52.

6. Mark AP, Hardeep SS. The Eradication of Polio-Progress and Challenges. N Engl J Med 2006; 355(24):2508-12.

7. Ali JM, Salah A, Shyam B, Padmamohan JK, Emadaldin E, Mahmoud M. et al. Fractional Doses of Inactivated Poliovirus Vaccine in Oman. N Engl J Med 2010; 362:2351-9. 